\title{
A loss-of-function mutation in Calmodulin2 gene affects pollen germination in Arabidopsis thaliana
}

\author{
Michela Landoni • Alessandra De Francesco • \\ Massimo Galbiati · Chiara Tonelli
}

Received: 30 December 2009/Accepted: 22 July 2010/Published online: 4 August 2010

(C) Springer Science+Business Media B.V. 2010

\begin{abstract}
Calmodulin (CAM) is an ubiquitous calcium binding protein whose function is to translate the signals, perceived as calcium concentration variations, into the appropriate cellular responses. In Arabidopsis thaliana there are 4 CAM isoforms which are highly similar, encoded by 7 genes, and one possible explanation proposed for the evolutionary conservation of the CAM gene family is that the different genes have acquired different functions so that they play possibly overlapping but non-identical roles. Here we report the characterization of the Arabidopsis mutant cam2-2, identified among the lines of the gene-trapping collection EXOTIC because of a distorted segregation of kanamycin resistance. Phenotypic analysis showed that in normal growth conditions cam2-2 plants were indistinguishable from the wild type while genetic analysis showed a reduced transmission of the cam $2-2$ allele through the male gametophyte and in vitro pollen germination revealed a reduced level of germination in comparison with the wild type. These results provide genetic evidence of the involvement of a $C A M$ gene in pollen germination and support the theory of functional diversification of the CAM gene family.
\end{abstract}

Keywords Arabidopsis thaliana - Calmodulin · GUS expression pattern · Loss-of-function mutant · Pollen germination

M. Landoni $(\bowtie)$ - A. De Francesco · M. Galbiati · C. Tonelli Dipartimento di Scienze Biomolecolari e Biotecnologie, Università degli Studi di Milano, via Celoria 26, 20133 Milan, Italy

e-mail: michela.landoni@unimi.it

\section{Introduction}

Calcium is an important second messenger involved in a variety of plant responses to biotic and abiotic stimuli, including pathogens, phytohormones, touch, drought, light, cold, heat, oxidative stress and developmental processes (Trewavas and Malhò 1998; Rudd and Franklin-Tong 2001). This multiplicity of stimuli results in rapid and transient $\mathrm{Ca}^{2+}$ fluctuations that modulate a cascade of cellular processes allowing the plant to respond to the changed environment. Because different stimuli trigger different and appropriate responses using the same second messenger, the cellular machinery responsible for the signal transduction must unequivocally identify the alteration of $\mathrm{Ca}^{2+}$ concentration produced by each specific stimulus. An important parameter defining the specificity of the $\mathrm{Ca}^{2+}$ signals is given by the so called " $\mathrm{Ca}^{2+}$ signature" that refers to the frequency, amplitude and spatial domain of $\mathrm{Ca}^{2+}$ release (Rudd and Franklin-Tong 2001; McAinsh and Pittman 2009). An additional level of specification is represented by the $\mathrm{Ca}^{2+}$ sensor that interprets the " $\mathrm{Ca}^{2+}$ signature" and by correctly interacting with the specific target protein determines, at the end, the appropriate cellular answer to the initial stimulus (Yang and Poovaiah 2003).

In plants three major classes of $\mathrm{Ca}^{2+}$ sensors have been characterized: calmodulin (CAM) and CAM-related proteins, calcium-dependent protein kinases (CDPK) and calcineurin B-like proteins (CBL) (Luan et al. 2002; Yang and Poovaiah 2003).

CAMs are the most conserved and the best known $\mathrm{Ca}^{2+}$ sensors. They have no enzymatic activity, but after $\mathrm{Ca}^{2+}$ binding they can regulate the activity of numerous target proteins involved in a variety of processes.

Typically CAMs contain 4 calcium binding sites, the EF-hands, that are helix-loop-helix motifs in which the two 
helices, named $\mathrm{E}$ and $\mathrm{F}$, are separated by the $\mathrm{Ca}^{2+}$ binding loop. Each EF-hand binds a single $\mathrm{Ca}^{2+}$ ion but the functional unit for CAM activity is a pair of EF-hand motifs, each of them organized in one of the two globular domains composing the CAM protein. In the presence of resting levels of $\mathrm{Ca}^{2+}(100 \mathrm{nM})$ the globular domains are in the socalled "closed" conformation but upon the binding of $\mathrm{Ca}^{2+}$ to CAM, triggered by the rapid elevation of $\mathrm{Ca}^{2+}$ caused by a stimulus, CAM proteins adopt the "open" conformation. This change results in the exposure of hydrophobic surfaces surrounded by negative charges, allowing the interaction with target proteins and the modulation of their activity (Luan et al. 2002).

Screening of cDNA libraries and bioinformatics tools have allowed the identification in plants of a large number of CAM-binding proteins (CAMBP) (Bouché et al. 2005) thus the high conservation of CAM sequences in eukaryotes (vertebrates' CAMs are identical and share 91\% amino acid identity with plant CAMs, McCormack and Braam 2003) could therefore result from the necessity to maintain a structure allowing the interaction with so many different target proteins (Ikura and Ames 2006).

In animals, only a few genes encoding one or a few CAM isoforms are present, conversely plants' genomes contain multiple $C A M$ genes encoding for several isoforms (Snedden and Fromm, 1998; Toutenhoofd and Strehler 2000).

In the Arabidopsis thaliana genome there are 7 CAM genes (Braam and Davis 1990; Ling et al. 1991; Perera and Zielinski 1992; Gawienowski et al. 1993; Zielinski 2002) and 50 CAM-like genes, encoding for proteins with EFhand motifs and at least $16 \%$ identical to CAM (McCormack and Braam 2003), confirming the complexity of $\mathrm{Ca}^{2+}$ sensing in higher plants. The $7 C A M$ genes encode for 4 different isoforms that show $96 \%$ of aminoacid identity with the highest divergence in the $3^{\prime}$ untranslated regions. In particular, with respect to the isoform CAM2/3/5, the isoform CAM1/4 differs by 5 amino acids, the isoform CAM6 differs by 2 amino acids and the isoform CAM7 differs by only 1 amino acid (McCormack et al. 2005).

Analyses of $C A M$ genes' sequence reveals that they are under strong selective pressure to maintain this high level of conservation and two possible explanations have been proposed: the necessity of a very high level of gene expression, accomplished by the simultaneous transcription of multiple genes, or the diversification of $C A M$ genes expression/regulation resulting in a different and specific function for each member of this gene family (McCormack et al. 2005).

Expression analyses of Arabidopsis CAM genes have revealed that even if these genes are expressed at high levels during all developmental stages, in every plant organ and with no significant changes in expression in response to different stimuli, each gene presents a specific profile, with genes encoding for identical proteins (CAMI/CAM4 and $C A M 2 / C A M 3 / C A M 5$ ) sharing the most similar patterns (McCormack et al. 2005).

The high level of CAM genes expression seems to support the hypothesis that the multiple genes are needed to satisfy the need for a high level of protein production. On the other hand the expression profiles are peculiar for each gene, leaving the possibility that the different $C A M$ genes have evolved different specialized functions. The determination of the functional role played by each $C A M$ gene appears to be a useful approach to shed light on the evolutionary meaning of this gene family and to clarify the functional relationship among genes encoding for the same isoform.

Increasing evidence supports a role for CAM proteins in plant development and in responses to biotic and abiotic stimuli (Ranty et al. 2006), but up to now only a few reports have presented experimental data about the physiological functions specifically played by the different members of this gene family. In particular in tobacco the three CAM isoforms NtCAM1, NtCAM3 and NtCAM13 have been shown to bind to the calcium-binding protein kinase NtCBK2 with different affinities and to modulate differently its enzymatic activity (Hua et al. 2003). In soybean the two divergent CAM isoforms CAM4 and CAM5, but not the other CAMs which are highly conserved, are involved in disease resistance responses (Heo et al. 1999), while the isoforms CAM1 and CAM4 differentially regulate the DNA binding activity of the AtMYB2 transcription factor (Yoo et al. 2005).

In Arabidopsis, only two members of this gene family have been functionally characterized, CAM3 (Zhang et al. 2009) and CAM7 (Kushwaha et al. 2008).

CAM7 is a transcriptional regulator involved in seedling development (Kushwaha et al. 2008), while CAM3 plays a key role in heat-shock signal transduction (Zhang et al. 2009).

Interestingly, Zhang and colleagues found that while the thermotolerance of the cam 3 knockout mutant was significantly reduced after the heat-shock treatment, cam2 seedlings showed a thermotolerance similar to the wild type (Zhang et al. 2009). Taken together these results suggest the existence of a functional diversification not only for genes encoding different CAM isoforms (CAM3 and $C A M 7$ ) but also for genes encoding for the same isoform (CAM3 and CAM2). The characterization of the physiological role played by the $C A M 2$ gene will give further support to this hypothesis of functional diversification of CAM family members.

Here we report on the characterization of the Arabidopsis mutant cam2-2, isolated in the EXOTIC gene trap collection (http://www.jic.bbsrc.ac.uk/hosting/exotic/main frameset.htm). In this line the modified transposon $D s G$, 
containing the GUS reporter gene, is inserted in the second exon of the CAM2 gene, causing the loss of function of this gene and resulting in a reporter gene activity mimicking the expression pattern of the tagged gene. The phenotypic and genetic characterization, guided by the specific expression pattern revealed by GUS analysis, suggests for the CAM2 gene a specific role in pollen germination and supports the theory of functional non-redundancy of $C A M$ genes.

\section{Materials and methods}

Plant material and growth conditions

The EXOTIC line GT_109750 (in this paper named cam2-2) (http://www.jic.bbsrc.ac.uk/hosting/exotic/mainframeset.htm) and the transgenic line cam2-2 35S::CAM2 were in the Arabidopsis thaliana ecotype Landsberg erecta (Ler). The T-DNA insertion knockout mutant of CAM3 (SALK_001357), obtained from the NASC European Arabidopsis Stock Center (Nottingham, GB) was in the Arabidopsis thaliana ecotype Columbia (Col) (Alonso et al. 2003).

The Arabidopsis seedlings were grown in soil at $22^{\circ} \mathrm{C}$ in a greenhouse with a 16-h-light/8-h-dark cycle at a fluorescent illumination of $100 \mu \mathrm{mol} \mathrm{m} \mathrm{m}^{-2} \mathrm{~s}^{-1}$ (100 micromoles of photons per square meter per second) and $60 \%$ humidity.

For in vitro growth, seeds were surface sterilized for $5 \mathrm{~min}$ in $100 \% \mathrm{v} / \mathrm{v}$ ethanol, followed by $5 \mathrm{~min}$ in $3 \%$ $\mathrm{NaClO}$, rinsed twice with sterile distilled water, stratified at $4^{\circ} \mathrm{C}$ in the dark for 3 days and then grown on MS medium (Sigma), with the appropriate antibiotic, if needed, in a growth chamber under the same conditions of light and temperature as those reported for the growth on soil.

Semi-quantitative reverse transcription polymerase reaction

Total RNA was extracted from 4 and 7 day old seedlings, 3 and 4 week old rosette leaves, 4 week old shoots and cauline leaves, floral buds, open flowers, green siliques, mature roots and pollen of wild type (Ler) plants (unless differently specified), as previously reported (van Tunen et al. 1988; Honys and Twell 2003).

For each sample, cDNA synthesis was performed by treating $5 \mu \mathrm{g}$ of Dnase-treated RNA with RT Superscript ${ }^{\mathrm{TM}}$ II (Invitrogen) according to the manufacturer's instructions.

For specific amplification of CAM2 transcript we used the forward primer PEX32 (5'- AACAATGGCGGATCAGCTC- $\left.3^{\prime}\right)$ and the reverse primer CAM2R (5'-AAGC CGAAGAAGTTGTTTCAATCC- $3^{\prime}$ ). The hybrid transcript CAM2-GUS was amplified with the forward CAM2 specific primer PEX32 together with the reverse GUS specific primer PEX59 (5'-CGCTGCGATGGATTCCGGCATAG- ${ }^{\prime}$ ).

The expression level of Ts $\beta 1$ gene, which encodes for the $\beta$ subunit of tryptophan synthase (Berlyn et al. 1989), was used as a quantitative control and Ts $\beta 1 \mathrm{~F} 1$ ( $5^{\prime}$-CTCA TGGCCGCCGGATCTTGA-3 ${ }^{\prime}$ ) and Ts $\beta 1 \mathrm{R} 1$ (5'-CTTGT CTCTCCATATCTTGAGCA- $3^{\prime}$ ) are the $T s \beta 1$ specific primers used. PCR reactions were carried out for 25 cycles to provide semi-quantitative data. The amplification products were separated on $1 \%$ agarose gel, blotted on Hybond $\mathrm{N}+$ positively charged nylon membrane (Amersham), hybridized with specific DIG-labelled probes and the signal was detected according to the manufacturer's instructions (DIG-High Prime Labelling and Detection Kit, Roche). The reverse transcription polymerase reaction (RT-PCR) analysis was repeated at least three times for each sample with similar results.

In vitro pollen germination

For the analysis of germination ratios and pollen tube elongation, the pollen was placed on a semi-solid medium (Hodgkin 1983) consisting of $0.01 \% \quad \mathrm{H}_{3} \mathrm{BO}_{3}, 0.08 \%$ $\mathrm{CaCl}_{2} \cdot 2 \mathrm{H}_{2} \mathrm{O}, 3 \%$ polyethylene glycol $6,000,20 \%$ sucrose and $0.7 \%$ Bacto-Agar, $\mathrm{pH}$ 6.0, and grown for 20-24 h, unless differently specified, in the semi-dark at $23^{\circ} \mathrm{C}$.

The analysis of pollen germination of cam2-2 $35 \mathrm{~S}:$ : CAM2 and Ler $35 S:: C A M 2$ plants were performed on 3 independent transgenic lines with similar results.

For statistical analysis of the germination ratios and pollen tube elongation, each experiment was repeated at least three times, for each sample we have collected pollen from more than 10 different plants, cultured in 4 different dishes, and for each dish at least 100 pollen grains were analyzed after in vitro growth. The results obtained were analyzed using the Student's $t$-test considering statistically significant the differences at $P<0.05$.

Histochemical analysis of pollen

To analyse pollen development, Alexander and DAPI staining were performed as previously reported (Alexander 1969; Howden et al. 1998). Each experiment was repeated at least three times and for each sample (wild type and cam2-2) we collected pollen from more than 10 different plants and for each plant at least 100 pollen grains were observed after staining.

Pollen tube accession to the ovules was analysed by Aniline staining performed on wild type and cam2-2 pistils pollinated with wild type or cam2-2 pollen, collected 4, 24 or $48 \mathrm{~h}$ after pollination and then stained as previously reported (Mori et al. 2006). At least 5 different pollinated 
pistils were collected and analysed for each cross and each time.

After the staining, pollen and pistils were analysed by IMAGE R.D1 microscope (Carl Zeiss) equipped with an AxioCam MRc1 digital camera and the AxioVision AC imaging software.

\section{GUS staining}

For GUS activity detection, seedlings and adult plant organs were incubated overnight at $37^{\circ} \mathrm{C}$ in the staining solution $\left(0.5 \mathrm{mg} \mathrm{ml}^{-1}\right.$ X-Gluc, $0.5 \mathrm{mM} \mathrm{FeCN,} 50 \mathrm{mM}$ sodium-phosphate buffer, $\mathrm{pH} 7,0.1 \%$ Triton $\mathrm{X} 100$ ), as previously reported (Sundaresan et al. 1995). After staining, the tissues were cleared in $100 \%$ ethanol and then observed with an Olympus SZX12 stereoscope, and pictures were taken with a digital camera, the Olympus $\mathrm{Ca}$ media C-7070 wide zoom.

Plasmid construct and Arabidopsis transformation

To obtain transgenic plants overexpressing the CAM2 gene, the wild type full length cDNA was amplified by RT-PCR. The forward and reverse primers were respectively: CAM2.1F (5'-AACAATGGCGGATCAGCTC-3') and CAM2.1R (5'-CG AAGAAGATGTTTCAATCCTCA-3'). The PCR amplified product was cloned in $\mathrm{pCR} 2.1 \mathrm{TOPO} \mathrm{TA}$ cloning vector (Invitrogen), after digestion with NotI/BamHI the fragment was subcloned in sense orientation into the polylinker region of the

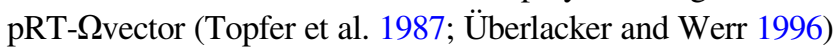
(downstream of the CaMV $35 \mathrm{~S}$ promoter). The construct obtained was digested with AscI and the expression cassette fragment was ligated into the AscI site of the pGPTV-HPT binary vector (Becker et al. 1992) sequenced and subsequently used to transform wild type (Ler) and cam2-2 plants, via Agrobacterium tumefaciens, strain GV3101, as previously reported (Clough and Bent 1998). Transgenic (T1) plants were selected on Murashige and Skoog medium containing $20 \mu \mathrm{g} \mathrm{ml}^{-1}$ Hygromycin B then were transferred into soil and allowed to self fertilize. T2 progeny were verified by PCR analysis using CAM2 specific primers and homozygous transgenic lines (T3) were used for further characterization.

Analysis of genetic transmission of cam2-2 mutation

The gametophytic transmission of the cam2-2 mutation was analysed by scoring the $D s G$ transmission, and in particular the kanamycin-resistance $\left(\operatorname{Kan}^{\mathrm{R}}\right)$ conferred by the nptII gene carried by this transposon (http://www. jic.ac.uk/met/handbook.pdf.hosting). We performed reciprocal backcrosses between wild type and cam2-2 plants: the seeds obtained from individual siliques were collected, germinated on agar-solidified MS medium containing Kan and the seedlings were scored for $\mathrm{Kan}^{\mathrm{S}}$ vs. $\mathrm{Kan}^{\mathrm{R}}$ phenotype. For statistical analysis we used the $\chi^{2}$ test.

\section{Results}

Isolation and molecular characterization of the cam2-2 line

As members of the EXOn Trapping Insertion Consotium (EXOTIC) we have contributed to the generation in Arabidopsis of about 30,000 gene trap lines composing the EXOTIC collection (http://www.jic.bbsrc.ac.uk/hosting/exotic/ mainframeset.htm).

This collection was generated by a gene trapping approach based on the $A c / D s$ transposon system of maize (Sundaresan et al. 1995): the different lines are characterized by the insertion of a modified $D s$ element ( $D s G$ ) containing the nptII gene, conferring kanamycin resistance and the GUS reporter gene. In this way a line in which the $D s G$ element is inserted in the correct orientation in the gene of interest, can be informative regarding both the function and the expression pattern of the disrupted gene (Galbiati et al. 2008).

During the selection of F2 transposant lines on kanamycin supplemented medium we have identified a line showing a distorted segregation ratio of kanamycin resistant $\left(\operatorname{Kan}^{\mathrm{R}}\right)$ vs kanamycin sensitive $\left(\mathrm{Kan}^{\mathrm{S}}\right)$ seedlings $\left(\operatorname{Kan}^{\mathrm{R}}: \mathrm{Kan}^{\mathrm{S}}=168: 83\right)$. The percentage of $\mathrm{Kan}^{\mathrm{R}}$ seedlings (\%R) was about $67 \%$, significantly different from the $75 \%$ expected for a correct 3:1 segregation ratio ( $P$ value of chi square test $<0.05)$.

The DNA sequence flanking the $D s G$ transposon was isolated by TAIL PCR technique (Liu et al. 1995) and BLAST analysis showed that the sequence of the TAILPCR product corresponds to the At $2 \mathrm{~g} 41110$ gene, encoding for the Calmodulin 2 (CAM2) protein.

Southern blot analysis detected the presence of only one $D s G$ insertion in the genome of this line (data not shown). PCR amplification and sequence analysis of the $D s G$ $C A M 2$ junction DNA, confirmed the insertion site of the $D s G$ element in the second exon of the CAM2 gene, in nucleotide position 468 downstream of the ATG codon (Fig. 1a) and showed the $8 \mathrm{bp}$ duplication typically produced by $D s$ insertion.

On the basis of these molecular data and considering the previously reported T-DNA insertional mutant cam2 (Zhang et al. 2009) we named this line calmodulin2-2 (cam2-2).

Sequence analysis also showed that the GUS reporter gene, carried by the $D s G$ element, was in the same orientation as the disrupted gene. This allowed the formation of a hybrid messenger, originated by the fusion of the $5^{\prime}$ 


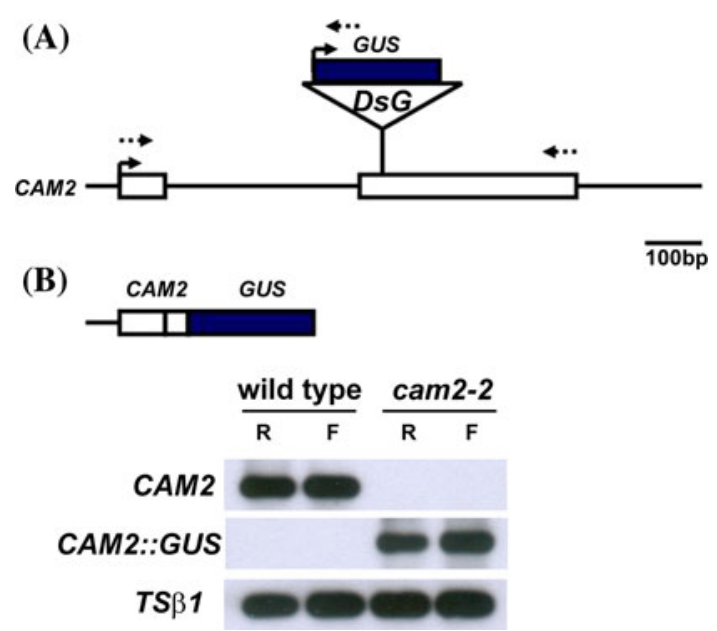

Fig. 1 Molecular characterization of the cam2-2 allele. a Diagrammatic representation of the site of $D s G$ insertion in the $C A M 2$ gene. Boxes represent the coding regions, the lines represent the $5^{\prime}$ and the $3^{\prime}$ untranslated regions and the intron, the bent arrows represent the position of the ATG start codon and the direction of transcription, the dotted arrows represent the position of the specific primers used for $C A M 2$ and $C A M 2:: G U S$ transcripts amplification in RT-PCR analysis. The $D s G$ element is represented with the only GUS gene (not drawn to scale). b Structure of the hybrid messenger $C A M 2:: G U S$. The GUS gene was not drawn to scale. c RT-PCR analysis of CAM2 gene and $C A M 2:: G U S$ hybrid expression in roots (R) and flowers (F) of wild type (Landsberg erecta genetic background) and cam $2-2$ plants. Ts $\beta 1$ expression levels were used as internal control

portion of the CAM2 mRNA (the first 104 nucleotides from the ATG) with the complete GUS transcript (Fig. 1b), whose presence has been confirmed by RNA gel blot, sequence analysis (data not shown) and RT-PCR analysis (Fig. 1c).

The deduced amino acid sequence of the hybrid CAM2GUS is composed by the first 34 amino acids of the CAM2 protein (corresponding to the first EF-hand motif truncated at the level of the F helix) fused to the complete aminoacidic sequence of the $\beta$-glucuronidase enzyme. This protein will therefore lack the functional domains for $\mathrm{Ca}^{2+}$ binding and for target interaction and thus not only will be impaired in its function as calmodulin but also will be unable to compete for $\mathrm{Ca}^{2+}$ and for specific target with other proteins. These data suggest that cam $2-2$ is a loss-offunction mutant.

Phenotypic analysis of the cam2-2 line

To try to obtain some clue about the specific function played by the CAM2 gene we performed a phenotypic analysis of cam2-2 plants. We analysed and compared with the corresponding wild type, the growth rate and morphology of whole plants and distinct organs of the cam2-2 line, during the development from seedling to adult plant, under normal growth conditions. In particular we measured the rosette diameter at 3 and 4 weeks after germination, the plant height at 3,4 and 5 weeks after germination, the primary root length at 4,7 and 14 days after germination on solid sterile medium, the silique length and the number of mature/unfertilized/aborted seeds contained per silique at maturity. No obvious alterations were found in cam2-2 line in comparison with the corresponding wild type (data not shown).

Expression analysis of $C A M 2$ gene

To examine the expression pattern of $C A M 2$ gene we performed semi-quantitative RT-PCR analysis on different tissues and organs of wild type plants at different developmental stages. The results obtained showed an ubiquitous and quite uniform expression of CAM2 gene in all the organs and developmental stages examined (Fig. 2a).

As previously reported, gene trap lines are useful tools for the identification of organ-, tissue- and cell-specific expression patterns (Galbiati et al. 2008), thus to try to obtain a more detailed picture of the CAM2 expression profile we analysed the localization of GUS activity in the cam2-2 line.

The histochemical assay, unlike the RT-PCR analysis, revealed a distinct and specific GUS expression pattern in the different organs and tissues analysed.

In vegetative organs, the $C A M 2$ gene was specifically expressed in the vascular tissue, in the different developmental stages analysed and in particular: in mature embryo (Fig. 2k), in seedlings 4 and 7 days old (Fig. 2a, b, respectively), in rosette leaves 3 and 4 weeks old (Fig. 2c, $\mathrm{d}$, respectively), in shoots (Fig. 2e), in cauline leaves (Fig. 2f) and in roots (Fig. 2j). In reproductive organs the GUS activity was detected in floral buds (Fig. $2 \mathrm{~g}, 1$ ), with the strongest signal corresponding to the youngest buds, and in open flowers (Fig. 2h, 1), with the higher expression in ovules. With the progression of the floral organs' development, the GUS staining became gradually less evident (Fig. 2l), in green siliques the GUS expression was detected only in the septum and in the funicle (Fig. 2i) and when the siliques turned yellow the latter was the only tissue showing GUS expression (Fig. 21).

Analysis of cam2-2 allele transmission through male and female gametophytes

We previously reported that the genetic analysis of heterozygous cam2-2/+ self-fertilized plants showed a reduced transmission to the progeny of the cam $2-2$ allele, carrying the kanamycin resistance. This result, supported by the GUS pattern showing a specific and elevated expression in floral organs, suggested a potential defect in male or 
Fig. 2 Expression analysis of the CAM2 gene. a Semiquantitative RT-PCR on wild type (Ler) plants.

b Histochemical localization of GUS activity on cam2-2 mutants. The same developmental stages and organs used for RT-PCR analysis were used also for GUS assay. (a) 4 day old seedling,

(b) 7 day old seedling,

(c) 3 week old rosette leaves,

(d) 4 week old rosette leaves,

(e) shoot, $(f)$ cauline leaves,

$(g)$ floral buds, $(h)$ open flower,

(i) green silique, $(j)$ root.

$(k)$ mature embryo and $(l)$ apical inflorescence were used only for GUS analysis
(A) $\begin{array}{llllllllll}\text { a } & \text { b } & \text { c } & \text { d } & \text { e } & \text { f } & \text { g } & \text { h } & \text { i } & \text { j }\end{array}$

CAM2

TS $\beta 1$

(B)
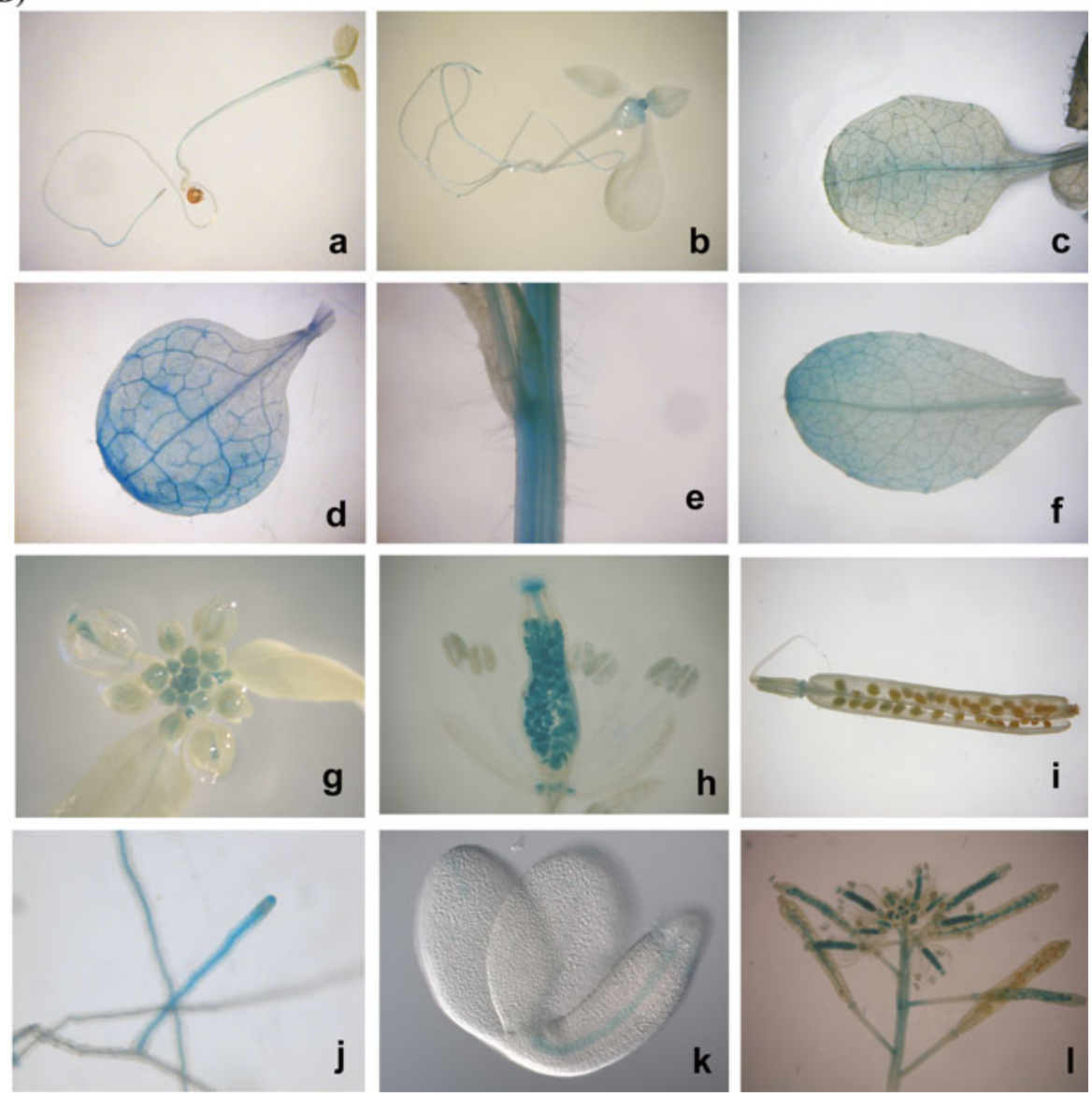

female gametophytes in cam2-2 plants, and thus an hypothetical role for $C A M 2$ gene in fertilization processes.

Hence to check the effect of the cam2-2 mutation on the success of male/female gametes we analysed the transmission efficiency of the kanamycin resistance in reciprocal backcrosses between plants heterozygous for the cam2-2 allele and wild type plants.

In Table 1 we report the results obtained: the segregation of $\operatorname{Kan}^{\mathrm{R}}$ vs. Kan ${ }^{\mathrm{S}}$ phenotypes, the percentage of $\operatorname{Kan}^{\mathrm{R}}$ seedlings $(\% \mathrm{R})$ and the transmission efficiency of the cam2-2 allele through male $\left(\mathrm{TE}_{\text {male }}\right)$ and through female $\left(\mathrm{TE}_{\text {female }}\right)$ gametes (Howden et al. 1998).

These data show that when pollen from heterozygous +/cam2-2 plants was used to pollinate wild type plants we observe a significant deviation from the expected 1:1 Mendelian ratio ( $P$ value of chi square test $<0.05$ ), with a loss of heterozygous progeny, while no significant deviation
Table 1 Transmission efficiency of the cam2-2 allele in reciprocal backrosses

\begin{tabular}{lllr}
\hline Backcross (female $\times$ male $)$ & $\mathrm{Kan}^{\mathrm{R}}: \mathrm{Kan}^{\mathrm{S}}$ & $\% \mathrm{R}^{\mathrm{a}}$ & $\mathrm{TE}^{\mathrm{b}}$ \\
\hline +/cam2-2 $\times$ wild type & $203: 198^{\mathrm{c}}$ & 50.6 & 102.5 \\
wild type $\times+/$ cam2-2 & $276: 355^{\mathrm{d}}$ & 43.7 & 77.7 \\
\hline
\end{tabular}

${ }^{\text {a }}$ Percentage of $\operatorname{Kan}^{\mathrm{R}}$ seedlings $\left(\operatorname{Kan}^{\mathrm{R}} / \operatorname{Kan}^{\mathrm{R}}+\operatorname{Kan}^{\mathrm{S}}\right)$

b Transmission efficiency of the DsG element (TE: $\operatorname{Kan}^{\mathrm{R}}$ / $\left.\mathrm{Kan}^{\mathrm{S}} \times 100\right)$

${ }^{c}$ No signicantly different from a 1:1 segregation ratio

d Significantly different from a 1:1 segregation ratio

was noted when wild type pollen was used to pollinate heterozygous plants.

The impaired transmission of the cam2-2 allele through male gametes indicates a competitive disadvantage of cam 2-2 pollen compared to the wild type and suggests a 
specific role for the CAM2 gene in male gametophyte function.

Effect of the cam2-2 mutation on pollen development and germination

To gain some insight about the possible function of the $C A M 2$ gene we then analysed more in detail the GUS expression pattern in during male gametophyte development (Fig. 3).

A strong GUS activity was detected in developing anthers until about stage 9 (Fig. 3a): after this stage, only a faint GUS staining was detectable in the anthers, as shown in an unfertilized flower at stage 10-11 (Fig. 3b) and in a fertilized flower at stage 15 (Fig. 3c), while after pollination a strong GUS activity appeared at the level of the stigma (Fig. 3d) and GUS activity was still present in germinating pollen grains (Fig. 3e; developmental stages as defined by Smyth et al. 1990).

To study a possible effect of the cam2-2 mutation on pollen development, we made histochemical analyses using DAPI staining, that allowed the visualization of the three pollen nuclei, and Alexander staining, which differentially stains aborted and non-aborted pollen (Fig. 4). These analyses showed that mitosis in cam2-2 pollen is normal, resulting in the three nuclei visualized by DAPI staining (Fig. 4a) and also that pollen vitality, revealed by Alexander staining, is identical in cam2-2 and in wild type
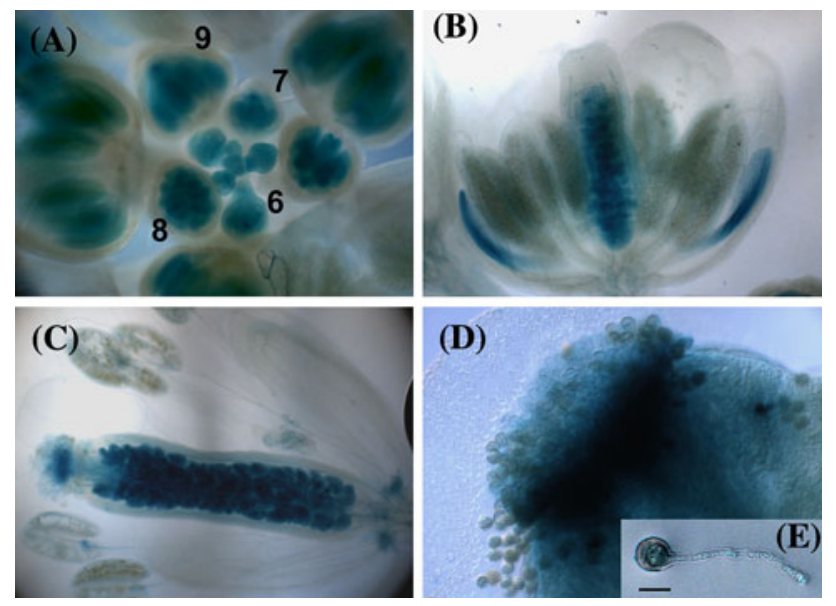

Fig. 3 Histochemical staining of GUS activity in cam2-2 floral organs. a Primary inflorescence showing intense GUS staining in all the organ primordia of the youngest floral buds. Starting from about stage 7, no GUS activity is present in sepals. Numbers on the floral buds indicate the developmental stages, identified as defined by Smyth et al. (1990). b Flower bud at stage 10-11, ovules and petals show intense GUS staining while only a faint staining is present in the anthers. c Open flower after pollination (stage 15), the higher GUS activity is present in the stigma, in the transmitting tissue and in developing seeds. d Pollinated pistil. e Germinating pollen on artificial medium. Scale bar, $20 \mu \mathrm{m}$

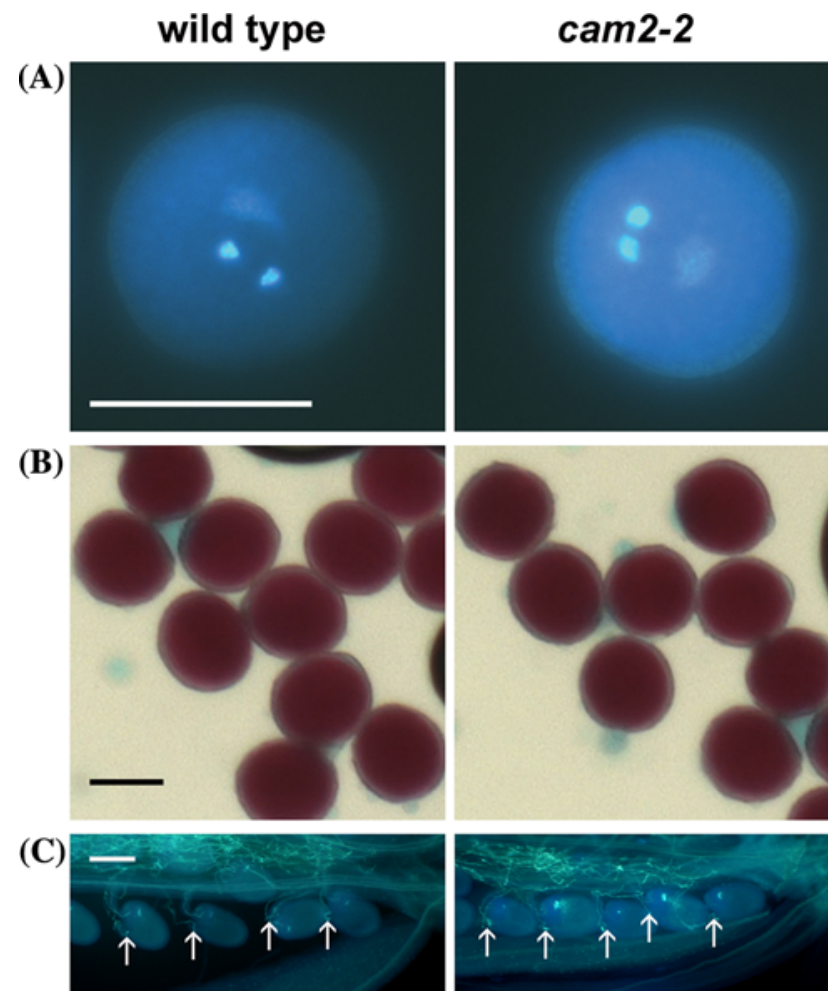

Fig. 4 Phenotype of cam2-2 mutant pollen grains compared to the wild type. a Analysis of nuclear constitution performed with DAPI staining. Scale bar, $20 \mu \mathrm{m}$. b Viability assay performed with Alexander staining. Scale bar, $20 \mu \mathrm{m}$. c Aniline blue staining of wild type and cam2-2 pollen tubes, $24 \mathrm{~h}$ after the pollination of wild type pistils. In both cases pollen tubes are able to germinate and grow though the transmitting tissue reaching and fertilizing of all the ovules present. Arrows indicate the pollen tubes accession to the ovules at the bottom of the silique. Scale bar, $100 \mu \mathrm{m}$

pollen (Fig. 4b). Microscopic analysis also showed that the cam2-2 pollen was indistinguishable from the wild type in shape and size.

To gain more insights into the possible effects of the cam2-2 mutation on the process of fertilization we verified the successful pollen tube accession to the ovules by in vivo analysis (Mori et al. 2006). We carried out Aniline blue staining, which allows the visualization of pollen tube growth through the stigma and the style, on wild type flowers after pollination with either wild type or cam2-2 pollen (Fig. 4c). The results showed that pollen tubes of both wild type and cam 2-2 mutants were able to grow and reach all the ovules, from the tip to the base of the pistil, to accomplish fertilization with no differences in growth rate or in efficiency of pollination (Fig. 4c). The same results were obtained when cam2-2 pistils were pollinated with wild type or cam2-2 pollen (data not shown).

The effect of the cam2-2 mutation on pollen development was also investigated by the analysis of in vitro pollen germination. Pollen was collected from wild type and cam 2-2 plants, grown in the same conditions, and after 
$24 \mathrm{~h}$ of growth on semi-solidified medium, pollen germination was analysed. The pollen grain was classified as germinated if the length of pollen tube was bigger that the pollen diameter, otherwise the pollen grain was classified as non-germinated.

The data obtained show that pollen germination was significantly reduced in the cam2-2 line compared with the wild type, with a reduction of nearly $30 \%$ (Fig. 5).

To verify if the mutant phenotype observed was the consequence of the $D s G$ insertion in the $C A M 2$ gene, we transformed cam2-2 plants with the full length CAM2 cDNA under the control of the CaMV 35S promoter. The germination efficiency was completely restored in cam $2-2$ $35 S:: C A M 2$ plants (this result was obtained by the analysis of 3 independent $\mathrm{T} 3$ trangenic lines as reported in "Materials and Methods") therefore the genetic complementation confirmed that the cam2-2 mutation was responsible for the observed reduction of germination efficiency (Fig. 5).

To further support these data we used the same construct to transform wild type plants. In the non mutated Ler background the expression of CAM2 cDNA under the control of the CaMV 35S promoter was associated, in each of the 3 independent transgenic lines analyzed, with a slight but significant increase $(P<0.05)$ of pollen

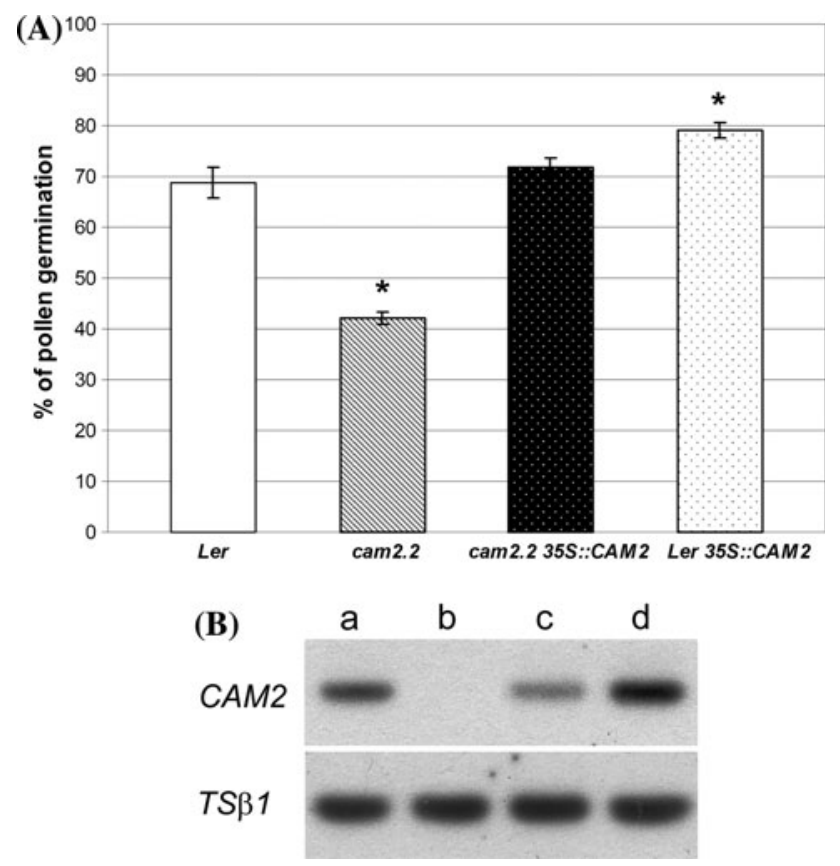

Fig. 5 a Percentage of in vitro pollen germination in wild type (Ler), cam2-2, cam2-2 35S::CAM2 and Ler 35S::CAM2 plants. Bars indicate standard error. Asterisks indicate statistically significant differences, in comparison with the wild type, as determined by Student's $t$-test $(P<0.05)$. b RT-PCR analysis of $C A M 2$ gene expression in wild type (Ler) (a), cam2-2 (b), cam2-2 35S::CAM2 (c) and Ler 35S::CAM2 (d) mature pollen. Ts $\beta 1$ expression levels were used as internal control germination, in comparison with the non transformed control (Fig. 5a).

Semi-quantitative RT-PCR analysis showed different expression levels of $C A M 2$ gene in pollen of wild type, cam2-2 35S::CAM2 and Ler 35S::CAM2 plants, with the lowest expression level in cam2-2 $35 S:: C A M 2$ pollen, suggesting that the CaMV 35S promoter was able to drive the expression of the cDNA in pollen but with a lower efficiency in comparison with the native promoter (Fig. 5b), as previously reported (Wilkinson et al. 1997).

In order to better characterize the defect of pollen germination displayed by the cam2-2 plants we performed a time course experiment: the germination efficiency and the pollen tube elongation of cam2-2 and wild type pollen were measured and compared after 1, 2, 4, 8, 12 and $24 \mathrm{~h}$ of in vitro germination. The results showed that while in each time analyzed the percentage of pollen germination of cam2-2 plants appeared to be significantly reduced in comparison with the wild type (Fig. 6a), no significant differences were found between mutant and wild type pollen tube elongation (Fig. 6b).

These results together with the data obtained by Aniline blue staining and the observed production by cam $2-2$ plants of a normal seed set, suggest that the cam2-2 pollen grains that were still able to germinate, could elongate the pollen tube to reach all the ovules and achieve the fertilization process normally, thus indicating that pollen germination was the only aspect of fertilization impaired by the cam2-2 mutation.

\section{Analysis of cam2cam3 double mutant phenotype}

Metaprofile analysis performed with the Genevestigator online search tool (https://www.genevestigator.com/gv/index. jsp; Zimmermann et al. 2004) showed that among the CAM genes, CAM3 displayed the highest expression level in pollen, so we tested if this gene could also play a role in pollen germination, in addition to its previously reported activity in heat shock signal transduction (Zhang et al. 2009).

Thus we crossed the cam2-2 and the cam 3 mutants to obtain the cam 2 cam 3 double mutant that in normal growth conditions was indistinguishable from the wild type and from the two single mutants cam2-2 and cam3.

The in vitro pollen germination of cam2cam 3 double mutant was then tested and compared with the single mutants and the corresponding wild type ecotype (Fig. 7).

The results showed that the germination efficiency of the cam3 single mutant was similar to the level observed in the corresponding wild type while the cam 2 cam 3 double mutant displayed a reduction of pollen germination that, considering that the two single mutants were in different ecotypes, was roughly comparable to the reduction 

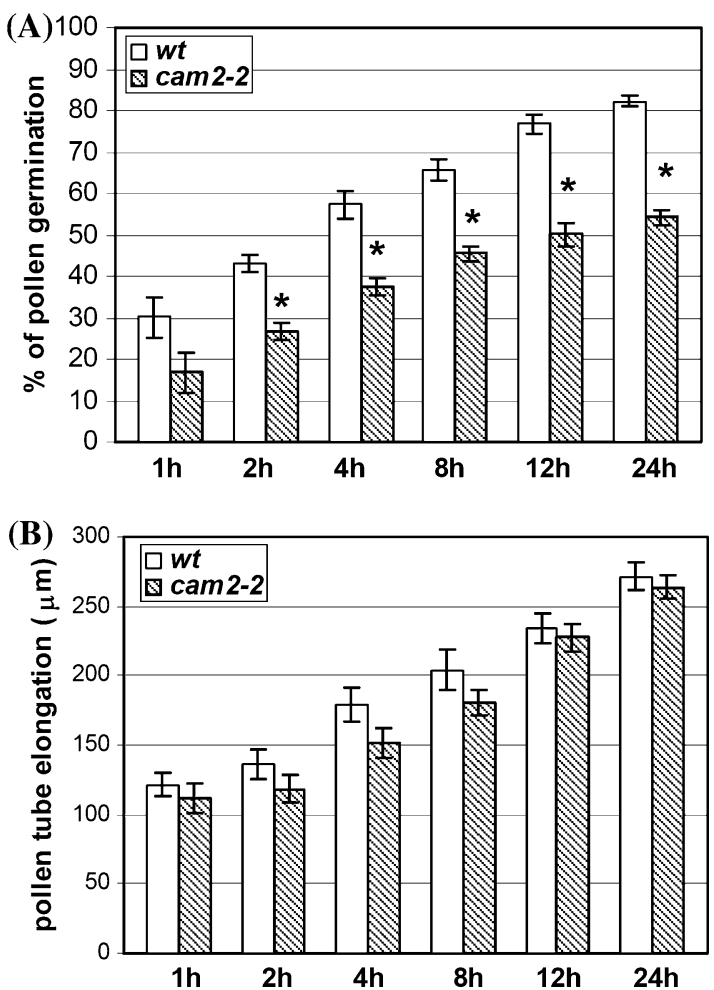

Fig. 6 Time-course of in vitro pollen germination a and pollen tube elongation $\mathbf{b}$ in wild type (Ler) and cam2-2 plants. Data were taken after 1, 2, 4, 8, 12 and $24 \mathrm{~h}$ of in vitro germination. Bars indicate standard error. Asterisks indicate statistically significant differences, in comparison with the wild type, as determined by Student's $t$-test $(P<0.05)$

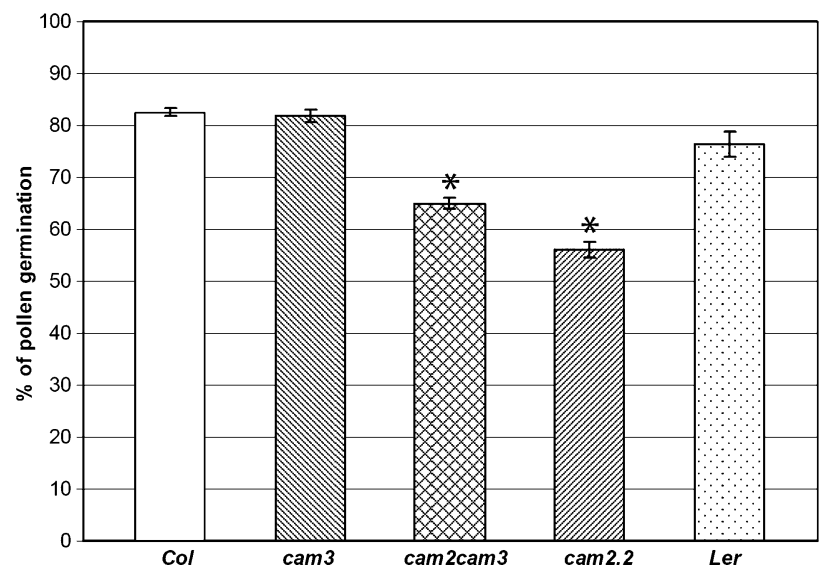

Fig. 7 Percentage of in vitro pollen germination in cam3, cam2-2, cam 2 cam 3 mutants plants and in the corresponding wild type ecotypes (Col for cam3 and Ler for cam2-2). Bars indicate standard error. Asterisks indicate statistically significant differences, in comparison with the wild type, as determined by Student's $t$-test $(P<0.05)$. For the double mutant cam2cam 3 the $t$-test was performed using both $\mathrm{Col}$ and Ler as control ecotype and in both tests the differences were statistically significant at $P<0.05$

observed for the cam2-2 single mutant. These data suggest that the $C A M 3$ gene is not functionally involved in pollen germination.

\section{Discussion}

Plant genomes contain a high number of paralogous genes derived from phenomenon of gene/chromosome/whole genome duplications, but although duplicated genes are generally predicted to degenerate and disappear, in plants a massive retention of paralogs is observed (Veitia 2005). Different theories have been proposed to explain this phenomenon: the subfunctionalization (the original gene function is split among the paralogs that have evolved specific expression patterns), the neofunctionalization (the paralogs gained new functions) or the need for the preservation of stoichiometry (for gene products involved in macromolecular complexes or pathways that require a dosage balance for proper function; Veitia 2005).

The Arabidopsis genome contains 7 CAM genes encoding for 4 different isoforms which are highly conserved, CAM1/CAM4, CAM2/CAM3/CAM5, CAM6 and CAM7 (McCormack and Braam 2003). Different non-allelic CAM genes have been identified both in plants and in animals but while in vertebrates there are few CAM genes encoding for a unique protein, in plants, there are generally present numerous CAM genes encoding different isoforms.

Analyses of the expression profiles revealed the existence of specific patterns for the CAM family members both in animals (Toutenhoofd and Strehler 2000) and in plants (Botella and Arteca 1994; Takezawa et al. 1995; Yang et al. 1998; Bergey and Ryan 1999; Heo et al. 1999; van der Luit et al. 1999; McCormack et al. 2005) therefore suggesting that these genes, differentially expressed, are necessary to ensure the spatial-temporal specificity needed for the $\mathrm{Ca}^{2+}$ signalling pathway (Toutenhoofd and Strehler 2000). The characterization of knockout mutants will help in elucidating the physiological role played by the different isoforms and by the different genes encoding for the same isoform.

Working on the generation of the EXOTIC collection of gene trap lines of Arabidopsis we found a transposant line characterized by a distorted segregation of the kanamycin resistance (http://www.jic.bbsrc.ac.uk/hosting/exotic/main frameset.htm). In this line the insertion of the $D s G$ element in the second exon of the CAM2 gene causes the loss of function of this gene and thus it appears to be a good candidate to investigate whether the functions played by the $C A M$ genes are overlapping or specific, and thus to find out why the evolutionary pressure maintains different $C A M$ genes (CAM2, CAM3 and CAM5) encoding for the same isoform.

The CAM2 functional analysis guided by the GUS expression pattern revealed a reduction of about $30 \%$ in germination efficiency of mutant pollen suggesting that the specific function of $C A M 2$ gene is required for pollen germination. 
The results presented, involving CAM2 in male gametophyte function, are not unexpected. It is known that $\mathrm{Ca}^{2+}$ plays an essential role during sexual plant reproduction (reviewed by Ge et al. 2007): it is generally necessary for pollen germination, it determines the directionality of tube elongation and is required for the fusion of gametes. A tipfocused gradient of cytosolic-free calcium is present in growing pollen tubes and CAM activity has been reported to display a similar tip-focused gradient (Rato et al. 2004). The pivotal role played by CAM in pollen tube growth regulation has been demonstrated by the use of CAM antagonists (Rato et al. 2004; Chen et al. 2009) or antiCAM antiserum (Ma et al. 1999) but to our knowledge, no direct genetic evidence for a specific role for a CAM gene in pollen function was yet presented. Our data, suggesting for CAM2 a role as a $\mathrm{Ca}^{2+}$ sensor specifically involved in pollen germination can shed some light on the first step of the signal transduction pathway that from the variation of $\mathrm{Ca}^{2+}$ concentration leads to pollen germination: the next step will be the identification and characterization of CAMBPs that specifically interact with CAM2.

In plants numerous CAMBPs have been identified, more than 20 plant-specific, and their high functional diversity reflects the multiplicity of signalling pathways involving CAM (Bouché et al. 2005). In particular, two different CAMBPs characterized in Arabidopsis, ACA9 (Autoinhibited $\mathrm{Ca}^{2+}$ ATPase) (Schiøtt et al. 2004) and NPG1 (No Pollen Germination1) (Golovkin and Reddy 2003), have been reported to be required for pollen germination and pollen tube growth. ACA9 gene is primarily expressed in pollen and mutations in this gene result in a semisterile phenotype, due to the functions played by this plasma membrane $\mathrm{Ca}^{2+}$ transporter as the key regulator of pollen tube growth and fertilization (Schiøtt et al. 2004). NPG1 is expressed only in pollen and its function is not necessary for male meiosis and pollen development but is essential for pollen germination (Golovkin and Reddy 2003).

No data are available about which of the CAM or CAMlike proteins interact with the identified CAMBPs, it may even be possible that the same CAMBP protein under different physiological conditions interacts with different CAM/CAM-like proteins, determining different cellular responses (Bouché et al. 2005). The identification of CAMBP specifically interacting with CAM2 will help in the understanding of the role played by this protein on pollen germination, to allow the discovery of the components of the $\mathrm{Ca}^{2+}$ signal transduction pathway directly involved in pollen germination.

During the process of fertilization the pollen, released from the anthers, reaches the compatible stigma, germinates, and, by rapid pollen tube growth through the female tissues, reaches and fertilizes the ovules. The functions played by the male gametophyte are very specialized and focused towards fertilization, the pollen transcriptome is therefore clearly reduced in comparison to that of vegetative tissues (Becker et al. 2003) and is characterized by the presence of genes committed to its specific functions in germination and pollen tube growth, i.e. signal transduction, cell wall biosynthesis and vesicle trafficking (Pina et al. 2005).

Different papers have reported on the characterization of the Arabidopsis pollen transcriptome (Honys and Twell 2004; Pina et al. 2005), and in particular Wang and colleagues analysed and compared the transcriptome during the different stages of pollen development, from mature pollen grain to pollen tube (Wang et al. 2008). The expression levels of $C A M / C M L$ families were found to be specifically changed, mainly up-regulated, during pollen germination or pollen tube growth, supporting their involvement in these processes. In particular the CAM7 gene appears to be up-regulated during pollen tube growth while $C A M 2$ and $C A M 3$ genes are highly expressed during both pollen germination and pollen tube growth. Proteome analysis of Arabidopsis mature pollen confirmed the presence of calmodulin (in particular CAM4 and CAM5), classified in the functional category of calcium binding and signalling proteins, corresponding to $5 \%$ of the proteins identified in pollen (Sheoran et al. 2006).

A very useful tool for a comparison of transcript abundance of selected genes in different plant organs is the meta-profile analysis performed by Genevestigator (Zimmermann et al. 2004).

Among the members of the CAM gene family (CAM6 probe was not available in this analysis) the most expressed genes in pollen are CAM3 and CAM2, and because these two genes encode for the same CAM isoform, together with CAM5, their high level of expression could suggest the necessity for pollen function of elevated levels of this isoform. However, the results we presented of in vitro pollen germination of cam 2 and cam 3 single mutants and cam2cam3 double mutants (Fig. 7) seemed to exclude a direct role for CAM3 in pollen germination and a functional redundancy of these two genes, encouraging the alternative hypothesis of a subfunctionalizion of CAM genes, further supported by the completely different expression pattern displayed by CAM5 gene, showing a very low expression level in pollen.

Nevertheless it cannot be excluded that $C A M$ genes encoding for a different isoform could share with CAM2 a role in pollen germination as, for example, CAM7 and CAM1 that displayed high levels of expression in pollen.

The characterization of single and multiple mutants in $C A M$ genes will clarify their functional relationships and in particular will reveal the extent of the specificity/redundancy of their roles.

Data supporting both the functional specificity and redundancy of $C A M$ genes are presented in two papers that 
report on the characterization of $C A M 3$ and $C A M 7$ genes (Zhang et al. 2009; Kushwaha et al. 2008).

Zhang and colleagues (2009) showed that while cam2, cam 3 and cam 4 mutants grown in normal conditions were indistinguishable from wild type, under heat shock conditions cam 3 was characterized by a significant impairment in thermotolerance. The authors proposed for this gene a key role in the $\mathrm{Ca}^{2+}$-CAM pathway of heat shock signal transduction and suggested that while the existence of functional redundancy among $C A M$ genes could explain the absence of phenotype alterations in normally grown cam mutants, the differential regulation of $C A M$ genes, even those encoding the same isoform, could explain the specific role played by $C A M 3$ under heat shock conditions (Zhang et al. 2009).

Our data suggesting the specific involvement of CAM2 but not of CAM3 in pollen germination, together with the observation that the cam 2 mutant did not display any differences in thermotolerance with respect to the wild type, unlike the cam3 mutant (Zhang et al. 2009), give further support to the idea of functional diversification of these two genes encoding for the same CAM isoform.

The functional characterization of $C A M 7$ revealed that this gene plays a role as transcriptional regulator of lightregulated genes promoting photomorphogenesis (Kushwaha et al. 2008).

As previously reported for CAM2 (Zhang et al. 2009 and this paper), and CAM3 (Zhang et al. 2009), mutations in the CAM7 gene also did not cause any altered morphology in normal growth conditions, and functional redundancy has been proposed by the authors as a possible explanation. But while for cam 2 and cam 3 mutants the absence of a mutant phenotype in normal growth conditions could be explained by the presence of 3 CAM genes (CAM2, CAM3 and CAM5) encoding for the same isoform, there is only one gene encoding for CAM7.

Experimental data show that the isoform CAM2/3/5, that differs from CAM7 by a single amino acid substitution, was not able to bind to the Z-box of light-regulated promoters as CAM7 specifically does, thus in this case the redundancy seems not to be among members of the CAM gene family, but might involve members of the same light transduction pathway or another signalling pathway working via CAM7 protein (Kushwaha et al. 2008).

For a complete functional dissection of the CAM gene family, it appears that it will be essential to characterize single and multiple cam mutants not only in normal growth conditions but also in the particular conditions in which the altered phenotypes of cam2, $\operatorname{cam} 3$ and $\operatorname{cam} 7$ mutants have been revealed. The only data thus far available are on the double mutants cam7cam3 (Kushwaha et al. 2008) and cam2cam3 (this paper) that did not display any altered phenotype in normal growth conditions.
In conclusion, our results show a significant reduction of pollen germination in the cam2-2 mutant, suggesting that the $C A M 2$ gene plays a specific function in this process, but the fully fertile phenotype displayed by cam $2-2$ mutants also supports the hypothesis of an, at least, partial functional redundancy, maybe involving $C A M 1$ and $C A M 7$ genes, highly expressed in pollen, and encoding for different CAM isoforms.

Our results together with the previously presented data regarding other cam mutants (Zhang et al. 2009; Kushwaha et al. 2008) may suggest that evolution has allowed the functional diversification of the different members of the $C A M$ gene family, in spite of the huge number and of the importance of the functions played by these genes, because in normal growth conditions the $C A M$ genes are, at least in part, functionally overlapping, so that a mutation in one $C A M$ gene does not result in the complete loss of the essential functions played by the CAM proteins.

\section{References}

Alexander MP (1969) Differential staining of aborted and nonaborted pollen. Stain Technol 44:117-122

Alonso JM, Stepanova AN, Leisse TJ et al (2003) Genome-wide insertional mutagenesis of Arabidopsis thaliana. Science 301: 653-657

Becker D, Kemper E, Schell J, Masterson R (1992) New plant binary vectors with selectable markers located proximal to the left T-DNA border. Plant Mol Biol 20:1195-1197

Becker JD, Boavida LC, Carneiro J, Haury M, Feijò JA (2003) Transcriptional profiling of Arabidopsis reveals the unique characteristics of pollen transcriptome. Plant Physiol 133: 713-725

Bergey DR, Ryan CA (1999) Wound- and systemin-inducible calmodulin gene expression in tomato leaves. Plant Mol Biol 40:815-823

Berlyn MB, Last RL, Fink GR (1989) A gene encoding the tryptophan synthase beta subunit of Arabidopsis thaliana. Proc Natl Acad Sci USA 86(12):4604-4608

Botella JR, Arteca RN (1994) Differential expression of two calmodulin genes in response to physical and chemical stimuli. Plant Mol Biol 24:757-766

Bouché N, Yellin A, Snedden WA, Fromm H (2005) Plant-specific calmodulin-binding proteins. Annu Rev Plant Biol 56:435-466

Braam J, Davis RW (1990) Rain-, wind-, and touch-induced expression of calmodulin and calmodulin related genes in Arabidopsis. Cell 60:357-364

Chen T, Wu X, Chen Y, Li X, Huang M, Zheng M, Baluska F, Samai J, Lin J (2009) Combined proteomic and cytological analysis of $\mathrm{Ca} 2+$-calmodulin regulation in Picea meyeri pollen tube growth. Plant Physiol 149:1111-1126

Clough SJ, Bent AF (1998) Floral dip: a simplified method for Agrobacterium-mediated transformation of Arabidopsis thaliana. Plant J 16:735-743

Galbiati M, Simoni L, Pavesi G, Cominelli E, Francia F, Vavasseur A, Nelson T, Bevan M, Tonelli C (2008) Gene trap lines identify Arabidopis genes expressed in stomatal guard cells. Plant $\mathrm{J}$ 53:750-762 
Gawienowski MC, Szymanski D, Perera IY, Zielinski RE (1993) Calmodulin isoforms in Arabidopsis encoded by multiple divergent mRNAs. Plant Mol Biol 22:215-225

Ge LL, Tian HQ, Russell SD (2007) Calcium function and distribution during fertilization in angiosperms. Am J Bot 94: 1046-1060

Golovkin M, Reddy ASN (2003) A calmodulin-binding protein from Arabidopsis has an essential role in pollen germination. Proc Natl Acad Sci USA 100:10558-10563

Heo WD, Lee SH, Kim MC, Chung WS, Chun HJ, Lee KJ, Park CY, Park HC, Choi JY, Cho MJ (1999) Involvement of specific calmodulin isoforms in salicylic acid-dependent activation of plant disease responses. Proc Natl Acad Sci USA 96:766-771

Hodgkin T (1983) A medium for germinating Brassica pollen in vitro. Crucif Newsl 8:62-63

Honys D, Twell D (2003) Comparative analysis of the Arabidopsis pollen transcriptome. Plant Physiol 132:640-665

Honys D, Twell D (2004) Transcriptome analysis of haploid male gametophyte development in Arabidopsis. Genome Biol 5:R85

Howden R, Park SK, Twell D (1998) The Arabidopsis thaliana gametophytic mutation gemini pollen1 disrupts microspore polarity, division asymmetry and pollen cell fate. Development 125:3789-3799

Hua W, Liang S, Lu YT (2003) A tobacco (Nicotiana tabacum) calmodulin-binding protein kinase, NtCBK2, is regulated differentially by calmodulin isoforms. Biochem J 376:291-302

Ikura M, Ames JB (2006) Genetic polymorphism and protein conformational plasticity in the calmodulin superfamily: two ways to promote multifunctionality. Proc Natl Acad Sci USA 103:1159-1164

Kushwaha R, Singh A, Chattopadhyay S (2008) Calmodulin7 plays an important role as transcriptional regulator in Arabidopsis seedling development. Plant Cell 20:1747-1759

Ling V, Perera I, Zielinski RE (1991) Primary structures of Arabidopsis calmodulin isoforms deduced from the sequences of cDNA clones. Plant Physiol 96:1196-1202

Liu YG, Mitsukawa N, Oosumi T, Whittier RF (1995) Efficient isolation and mapping of Arabidopsis thaliana T-DNA insert by thermal asymmetric interlaced PCR. Plant J 8:457-463

Luan S, Kudla J, Rodriguez-Concepcion M, Yalovsky S, Gruissem W (2002) Calmodulins and Calcineurin B-like proteins: calcium sensors for specific signal response coupling in plants. Plant Cell S389-S400

Ma L, Xu X, Cui S, Sun D (1999) The presence of a heterotrimeric G protein and its role in signal transduction of extracellular calmodulin in pollen germination and tube growth. Plant Cell 11: 1351-1363

McAinsh MR, Pittman JK (2009) Shaping the calcium signature. New Phytol 181:275-294

McCormack E, Braam J (2003) Calmodulins and related potential calcium sensors of Arabidopsis. New Phytol 159:585-598

McCormack E, Tsai YC, Braam J (2005) Handling calcium signaling: Arabidopsis CaMs and CMLs. Trends Plant Sci 10:383-389

Mori T, Kuroiva H, Higashiyama T, Kuroiva T (2006) GENERATIVE CELL SPECIFIC 1 is essential for angiosperm fertilization. Nat Cell Biol 8:64-71

Perera I, Zielinski RE (1992) Structure and expression of the Arabidopsis CaM-3 calmodulin gene. Plant Mol Biol 19: 649-664

Pina C, Pinto F, Feijo JA, Becker JD (2005) Gene family analysis of the Arabidopsis pollen transcriptome reveals biological implications for cell growth, division control, and gene expression regulation. Plant Physiol 138:744-756

Ranty B, Aldon D, Galaud JP (2006) Plant calmodulins and calmodulin-related proteins. Plant Signal Behav 1:96-104
Rato C, Monteiro D, Hepler PK, Malho R (2004) Calmodulin activity and cAMP signalling modulate growth and apical secretion in pollen tubes. Plant J 38:887-897

Rudd JJ, Franklin-Tong VE (2001) Unravelling response-specificity in $\mathrm{Ca}^{2+}$ signalling pathways in plant cells. New Phytol 15:7-33

Schiøtt M, Romanowsky SM, Baekgaard L, Jakobsen MK, Palmgren MG, Harper JF (2004) A plant plasma membrane $\mathrm{Ca}^{2+}$ pump is required for normal pollen tube growth and fertilization. Proc Natl Acad Sci USA 101:9502-9507

Sheoran IS, Sproule KA, Olson DJH, Ross ARS, Sawhney VK (2006) Proteome profile and functional classification of proteins in Arabidopsis thaliana (Landsberg erecta) mature pollen. Sex Plant Reprod 19:185-196

Smyth DR, Bowman JL, Meyerowitz EM (1990) Early flower development in Arabidopsis. Plant Cell 2:755-767

Snedden WA, Fromm H (1998) Calmodulin, calmodulin-related proteins and plant responses to the environment. Trends Plant Sci 3:299-304

Sundaresan V, Springer P, Volpe T, Haward S, Jones JD, Dean C, Ma H, Martienssen R (1995) Patterns of gene action in plant development revealed by enhancer trap and gene trap transposable elements. Genes Dev 9:1797-1810

Takezawa D, Liu ZH, An G, Poovaiah BW (1995) Calmodulin gene family in potato: developmental and touch-induced expression of the mRNA encoding a novel isoform. Plant Mol Biol 27: 693-703

Topfer R, Matzeit V, Gronenborn B, Steinbiss HH (1987) A set of plant expression vectors for transcriptional and translational fusion. Nucleic Acids Res 15:5890

Toutenhoofd SL, Strehler EE (2000) The calmodulin multigene family as a unique case of genetic redundancy: multiple levels of regulation to provide spatial and temporal control of calmodulin pools? Cell Calcium 28:83-96

Trewavas AJ, Malhò R (1998) $\mathrm{Ca}^{2+}$ signalling in plant cells: the big network!. Curr Opin Plant Biol 1:428-433

Überlacker B, Werr W (1996) Vectors with rare-cutter restriction enzyme sites for expression of open reading frames in transgenic plants. Mol Breed 2:293-295

van der Luit AH, Olivari C, Haley A, Knight MR, Trewavas AJ (1999) Distinct calcium signaling pathways regulate calmodulin gene expression in tobacco. Plant Physiol 121:705-714

van Tunen AJ, Koes RE, Spelt CE, van der Krol AR, Stuitje AR, Mol JN (1988) Cloning of the two chalcone flavanone isomerase genes from Petunia hybrida: coordinate, light-regulated and differential expression of flavonoid genes. EMBO J 7:1257-1263

Veitia R (2005) Paralogs in polyploids: one for all and all for one? Plant Cell 17:4-11

Wang Y, Zang WZ, Song LF, Zou JJ, Su Z, Wu WH (2008) Transcriptome analyses show changes in gene expression to accompany pollen germination and tube growth in Arabidopsis. Plant Physiol 148:1201-1211

Wilkinson JE, Twell D, Lindsey K (1997) Activities of CaMV 35S and nos promoters in pollen: implications for field release of transgenic plants. J Exp Bot 48:265-275

Yang T, Poovaiah BW (2003) Calcium/calmodulin-mediated signal network in plants. Trends Plant Sci 8:505-512

Yang T, Lev-Yadun S, Feldman M, Fromm H (1998) Developmentally regulated organ-, tissue-, and cell-specific expression of calmodulin genes in common wheat. Plant Mol Biol 37:109-120

Yoo JH, Park CY, Kim JC, Heo WD, Cheong MS, Park HC, Kim MC, Moon BC, Choi MS, Kang YH, Lee JH, Kim HS, Lee SM, Yoon HW, Lim CO, Yun DJ, Lee SY, Chung WS, Cho MJ (2005) Direct interaction of a divergent $\mathrm{CaM}$ isoform and the transcription factor, MYB2, enhances salt tolerance in Arabidopsis. J Biol Chem 280:3697-3706 
Zhang W, Zhou RG, Gao YJ, Zheng SZ, Xu P, Zhang SQ, Sun DY (2009) Molecular and genetic evidence for the key role of AtCam3 in heat-shock signal transduction in Arabidopsis thaliana. Plant Physiol 149:1773-1784

Zielinski RE (2002) Characterization of three new members of Arabidopsis thaliana calmodulin gene family: conserved and highly diverged members of gene family functionally complement a yeast calmodulin null. Planta 214:446-455

Zimmermann P, Hirsch-Hoffmann M, Henning L, Gruissem W (2004) GENEVESTIGATOR: Arabidopsis microarray database and analysis toolbox. Plant Physiol 136:2621-2632 